\title{
XXV. \\ Ueber das Vorkommen von Harnstoff im Muskel der Säugethiere. \\ Von
}

M. Nencki und A. Kowarski.

Die Angaben über das Vorkommen des Harnstoffes im Muskel der Sängethiere sind seit vielen Jahren wie die Nachrichten über die Seeschlange. Im Jahre 1878 bestimmte Pi $\operatorname{car} \mathbf{d}^{1}$ ) die Menge Stickstoff, welche aus den Organextracten durch $\mathrm{NaOBr}$ entwickelt werden und berechnet aus dem gefundenen Stickstoff den Harnstoffgehalt. Danach findet er, dass Muskel den grössten Harnstoffgehalt hat. Die Angabe Demant's ${ }^{2}$ ), welcher Harnstoff als salpetersauren Harnstoff aus Pferdefleisch dargestellt haben wollte, sowie die andern positiven Angaben, hat sein Lehrer Hoppe-Seyler ${ }^{3}$ ) später dementirt. Im Jahre 1884 bestimmte Hayeraft ${ }^{4}$ ) den Harnstoffgehalt im Muskel bei Ruhe und Arbeit, nachdem er sich überzeugte, dass Harnstoff im Muskel vorhanden ist; im Jahre 1889 finden Gréhaut und Quinquaud 5 ) in $100 \mathrm{~g}$ Muskel 37,8 resp. 107,3 mg Harnstoff, wiederum aus dem entwickelten Stickstoff in dem Fleischanszuge berechnet.

Anlässlich der Untersuchung über die Harnstoffbildung bei den Säugethieren, wollten wir uns in dieser Frage Gewissheit verschaffen und haben in einem Versuche 2,5 Kilo frische Hundemuskel nach dem Verfahren von v. Schröder auf Harnstoff verarbeitet, ohne eine Spur Harnstoff isoliren zu können. In einem zweiten Versuche wurde der wässerige Auszug von einem Kilo Hundemuskel mit Posphorwolframsäure und Salzsäure vollkommen ausgefällt. Nach Entfernung der überschüssigen Phosphorwolframsäure mit pulverigem Kalk wurde das alkalische Filtrat auf ein Liter gebracht

1) Maly's Jahresbericht. Bd. VIII. S. 262.

2) Ebenda. Bd. X. S. 351.

3) Dessen Lehrbuch der physiologischen Chemie. S. 644.

4) Maly's Jahresbericht. 1884. S. 540 .

5) Compt. rend. T. CVIII. p. 1092. 
und davon in 2 Proben je 50 resp. $100 \mathrm{~g}$ der Lösung mit $10 \mathrm{~g}$ krystallisirter Phosphorsäure eingedampft, sodann 3 Stunden anf 240-270 erhitzt und durch Destillation mit Natronlange das entstandene Ammoniak bestimmt. Wir erhielten für $100 \mathrm{eem}$ der Lösung: 72,6 resp. $73 \mathrm{mg}$ Ammoniak. Die restirenden $850 \mathrm{cem}$ der Lösung wurden mit oxalsaurem Ammoniak genau ausgefällt, von Kalkoxalat filtrirt, zur Trockne auf dem Wasserbade verdunstet und wiederholt mit absolutem Alkohol ausgezogen. Der alkoholische Auszug, zur Trockne verdunstet, wurde von Nenem mit absolutem Alkohol und etwas Aether in der Kälte aufgenommen, von ungelöstem Salmiak filtrirt und von neuem verdunstet. Aus dem syrupigen Rückstande,

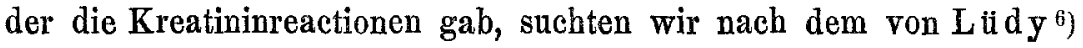
ausgearbeiteten Verfahren, Harnstoff nachzuweisen. Das Verfahren beruht auf dem Principe, dass eine alkoholische Harnstofflösung mit alkoholischer Lösung von ortho-Nitrobenzaldehyd, auf dem Wasserbade verdunstet, in

$$
\text { ortho-Nitrobenzylidendiureïd }=\mathrm{C}_{6} \mathrm{H}_{4}\left(\mathrm{NO}_{2}\right) \mathrm{CH}: \begin{aligned}
& \mathrm{NH} \cdot \mathrm{CO} \cdot \mathrm{NH}_{2} \\
& \mathrm{NH} . \mathrm{CO} \cdot \mathrm{NH}_{2}
\end{aligned}
$$

verwandelt wird. Das ortho-Nitrobenzylidendiureid ist ein weisslicher, krystallinischer Körper, der bei $200^{\circ}$ schmilzt, in Wasser und Alkohol sehr wenig löslich ist und durch Erwärmen mit verdünnten Mineralsäuren leicht in Harnstoff und ortho-Nitrobenzaldehyd zerfällt. Der letztere Körper, in alkoholischer Lösung; mit Phenylhydrazin versetzt, färbt sich sofort schön roth, indem er das in scharlachrothen, prismatischen Nadeln krystallisirende Hydrazon $=\mathrm{C}_{6} \mathrm{H}_{4}\left(\mathrm{NO}_{2}\right) \mathrm{CH}: \mathrm{N} . \mathrm{NH} \cdot \mathrm{C}_{6} \mathrm{H}_{5}$ giebt. Zum Nachweis des Harnstoffs wird der alkoholische Auszug des syrupartigen Verdampfungsrückstandes, aus der eventuell Harnstoff enthaltenden Flüssigkeit mit einer alkoholischen Lösung von ortho-Nitrobenzaldebyd versetzt. Nitrobenzaldehyd muss in solcher Quantität vorhanden sein, dass aller Harnstoff, der muthmaasslich angenommen werden darf, in Reaction treten kann. Die alkoholische Lösung wird auf dem Wasserbade zur Trockne eingedampft, hernach mit Alkohol übergossen, kurze Zeit erwärmt und der Alkohol abgegossen. Dies wird 2-3 mal wiederholt, $d . \mathbf{h}$. so lange, bis alle in Alkohol löslichen Stoffe wieder entfernt sind und der Alkohol mit Phenylhydrazinlösung keine Farbenreaction mehr zeigt, also auch uiberschüssig zugesetztes Nitrobenzaldehyd verschwunden ist. War Harnstoff vorhanden, so hinterbleibt das Condensationsproduct Nitrobenzylidendinreïd - als gelbweisser, pulveriger Körper, der sehr

1) Wiener Akademie-Berichte. Mathem,-naturw. Classe. Bd. XCVIII und Wiener Monatshefte f. Chemie. 1889. 
intensiv an den Wänden der Porzellanschaale haftet und infolge dessen auch bei minimalen Mengen sehr leicht wabrgenommen werden kann. Nunmehr wird der Rückstand mit wenig verdünnter Lösung von salzsaurem Phenylhydrazin übergossen, mit circa 5 bis 10 Tropfen einer 10 procentigen Schwefelsäure versetzt tund zum Sieden erhitzt. War der Rückstand wirklich Nitrobenzylidendiureïd, so wird sich die Flüssigkeit sogleich röthen, infolge Bildung des schon erwähnten, farbigen Körpers - des Phenylhydrazons des ortho-Nitrobenzaldehyds. Mittelst dieser Reaction können $5 \mathrm{mg}$ Harnstoff noch gut nachgewiesen werden, ja sogar in einer Lösung die nur $1 \mathrm{mg}$ Harnstoff enthielt, war dessen Anwesenheit noch zu constatiren.

Es gelang aber nicht auch mittelst dieser Reaction aus dem wässrigen Auszuge von $850 \mathrm{~g}$ Hundemuskel Harnstoff nachzuweisen. -

Man könnte immerhin noch einwenden, der Harnstoffgehalt sei so gering, dass bei der von uns verwendeten Muskelmenge der Nachweis nicht möglich sei. Wir haben daher $450 \mathrm{~g}$ Liebig'schen Fleisehextractes in kaltem Wasser gelöst und mit 10 Proc. Phosphorwolframsäure, der 1/10 ihres Volumens Salzsäure zagesetzt war, vollkommen ausgefällt. Es waren dazu über 2,5 Kilo krystallisirter Phosphorwolframsäure nöthig. Aus den Versuchen von Gumlich 1) und $\mathrm{Schöndorff}{ }^{2}$ ) wissen wir, dass Harnstoff in 1-3 procentiger Lösung durch Phosphorwolframsäure anch dann nicht gefällt wird, wenn die Lösung Ammoniaksalze und andere stiekstoffhaltige Bestandtheile des Harns enthält. - Der Phosphorwolframsäureniederschlag wurde abfiltrit, ausgewaschen, durch pulverigen Kalk alkalisch gemacht, filtrirt und aus dem Filtrate der Kalk durch oxalsaures Ammon ansgefällt. Das Filtrat vom Kalkoxalat wurde auf dem Wasserbade verdunstet und zur Trennung vom ausgeschiedenen Salmiak mit absolutem Al. kohol extrahirt. Der alkoholische Auszug von neuem verdunstet und mit Alkoholäther behandelt, wobei noch etwas Salmiak ungelöst zurückblieb. Jetzt wurde der nach Verdunsten des Alkoholäthers hinterbliebene syrupige Rüekstand mit Wasser aufgenommen und mit Zinkoxyd zum Sieden erhitzt, wobei ziemlich viel Ammoniak entwich, von dem noch gelösten Salmiak herrührend. Aus dem Filtrate von überschïssigem Zinkoxyd schieden sich nach dem Verdunsten etwa auf die Hälfte des ursprünglichen Volumens, Krystalldrusen $a b$, welche dem Aussehen nach Kreatininchlorzink waren. Die Krystalle wurden abfiltrirt, mit etwas Wasser nachgewaschen und ergaben nach dem Trocknen folgende Zahlen: 0,4931 $\mathrm{g}$ der Substanz, in schwach

1) Zeitschr. f. phys. Chemie. Bd. XVII. S. 13.

2) Pflüger's Archiv. Bd. LIV. S. 426. 
salzsaurem Wasser gelöst, und in der Siedhitze mit Soda im Ueberschuss gefällt gaben $0,1084 \mathrm{~g} \mathrm{ZnO}=17,64 \mathrm{Zn}$. Die Formel $\left(\mathrm{C}_{4} \mathrm{H}_{i} \mathrm{~N}_{3} \mathrm{O}\right)_{2} \mathrm{ZnCl}_{2}$ verlangt 17,95 Proc. $\mathrm{Zn}$.

Das Filtrat von dem ausgeschiedenen Kreatinchlorzink wurde mit Schwefelwasserstoff zerlegt, vom Schwefelzink abfiltrirt, dureh einen Luftstrom vom Schwefelwasserstoff befreit und mit Aether wiederholt extrahirt. In den Aether ging in reichlichen Mengen rechtsMilchsäure über, welche in das Zinksalz verwandelt, durch Krystallwasser- und Zinkbestimmung identificirt wurde. Die von Milchsäure befreite Lösung wurde mit Barytwasser alkalisch gemacht, das überschüssige Baryum durch Kohlensäure entfernt, filtrirt und auf dem Wasserbade verdunstet. Der syrupige Rückstand enthielt noch reichlich Kreatin und Kreatinin, die theilweise auskrystallisirten. Die Mutterlauge der Krystalle gab jedoch, sowohl mit Quecksilberchlorid als auch mit Pikrinsäure krystallinische Niederschläge der entsprechenden Kreatininverbindung. Nach möglichster Entfernung des Kreatin and Kreatinins extrahirten wir die syrupösen Rtïckstände mit Alkohol und Aether und suchten in den alkoholischen Ausziugen Harnstoff nachzuweisen. Die Proben mit ortho-Nitrobenzaldehyd fielen negativ aus. Wurde der alkoholische Rückstand in Wasser gelöst und mit Quecksilbernitrat versetzt, so entstand ein geringer weisser Niederschlag, der sich im überschüssigen Quecksilbernitrat wieder löste und der sicher nicht die Harnstoffquecksilberverbindung sein konnte, da dieser Niederschlag mit Schwefelwasserstoff zersetzt, dann nach Verjagen des Schwefelwasserstoffs durch die Luft, Behandeln mit Baryt und Kohlensäure, ein Filtrat gab, das mit einer Lösung von Diphenylamin in Schwefelsäure nicht die geringste Blaufärbung, d. h. Reaction auf Salpetersäure gab; während alle die Harnstoffverbindungen mit Quecksilbernitrat Salpetersäure in ihrem Molekïl enthalten. Wir kommen daher zu dem Schluss, dass die Muskel der Säugethiere keine, mit unseren doch sehr empfindlichen Reagentien nachweisbare Harstoffmenge, enthalten.

Nach den Bestimmungen von $\mathrm{H}$ ofmeister ${ }^{1}$ ) wird Kreatin in verdünnter, mit Salzsäure versetzter Lösung durch Phosphorwolframsäure nicht gefällt. Kreatinin giebt mit Phosphorwolframsäure ein krystallinisches Salz schon in Verdünnungen von 1:12,000. Da wir wegen der grossen Masse des Niederschlages in den Filtraten sammt Waschwasser mehr als 15 Liter Flüssigkeit hatten, so war jedenfalls ein Theil des von uns gefundenen Kreatinins schon als solches im

1) Zeitschrift f. phys. Chemie. Bd. V. S. 73. 
Fleichextract vorhanden. Ein anderer dürfte von dem gelösten Kreatin herribren. Die syrupigen, von uns erhaltenen kreatininhaltigen Riickstände, enthalten sicher noch andere, stickstoffhaltige Körper, da die Menge dieses Syrups ganz erheblich ist. Die kürzlich publicirten Untersuchungen von M. Siegfried ${ }^{1}$ ) über Fleischsäure und die von F. Ha iser ${ }^{2}$ ) über Inosinsäure bringen uns ganz neue Gesichtspunkte über den Stoffwechsel des Muskels und dürften fortgesetzte Untersuchungen der Extractivstoffe des Fleisches noch manche interessante Thatsache nach dieser Richtung hin bringen. Xanthinbasen, oder richtiger durch ammoniakalisches Silber fällbare Substanzen, enthält das Filtrat von Phosphorwolframsïureniederschlag nicht. Das nach Ausfällen mit Phosphorwolframsäure aus dem Filtrate durch Erhitzen mit Phosphorsäure auf $240-270^{\circ}$ erhaltene Ammoniak, dürfte hauptsächlich vom Kreatin des Fleisches herstammen. Wenn wirklich Harnstoff im Muskel der Säugethiere vorhanden wäre, so sehen wir nicht ein, warum er nicht daraus darstellbar sein sollte; da er doch z. B. aus dem Muskel der Rochen und Haie nach gleichen Methoden leicht dargestellt werden kann. Ebenso haben wir schon aus $100 \mathrm{~g}$ Hundeblut, nach vorausgegangener Fällung mit Phosphorwolframsäure, Harnstoff als ortho-Nitrobenzylidendiureïd dargestellt. Der aus der alkoholisehen Lösung erhaltene Körper schmolz bei $203^{\circ}$ und gab nach dem Kochen mit verdünnter Schwefelsäure und Zusatz von Phenylhydrazin das Phenylhydrazon des ortho-Nitrobenzaldebyds in Form von rothen Krystallnadeln.

1) Archiv f. Anat. u. Physiol. 1894. Physiol. Abth. S. 401.

2) Wiener Monatshefte für Chemie. Bd. XVI. S. 190. 1895. 